\title{
Aging amplifies multiple phenotypic defects in mice with zinc transporter Zip14 (Slc39a14) deletion
}

Tolunay Beker Aydemir ${ }^{\mathrm{a}}$, Catalina Troche ${ }^{\mathrm{a}}$, Jinhee Kim ${ }^{\mathrm{a}}$, Min-Hyun Kim ${ }^{\mathrm{a}}$, Oriana Y. Teran ${ }^{\mathrm{a}}$, Christiaan Leeuwenburgh ${ }^{b}$, Robert J. Cousins ${ }^{a}$

${ }^{a}$ Food Science and Human Nutrition Department

Center for Nutritional Sciences

College of Agriculture and Life Sciences

University of Florida

Gainesville, Florida

${ }^{b}$ Department of Aging and Geriatric Research

and Institute on Aging

College of Medicine

University of Florida

Gainesville, Florida

Corresponding Author:

Dr. Robert J. Cousins

352-392-2133

cousins@ufl.edu

Keywords:

Inflammation, Sarcopenia, Signaling pathways, Interleukin-6, bone, growth 
6

\section{Abstract}

Inflammation and zinc dyshomeostasis are two common hallmarks of aging. A major zinc transporter ZIP14 (slc39a14) is upregulated by proinflammatory stimuli, e.g. interleukin-6. We have evaluated the influence of age on the Zip14 KO phenotype using wild-type (WT) and Zip14 knockout (KO) mice. Aging produced a major increase in serum IL-6 concentrations that was dramatically augmented in the Zip $14 \mathrm{KO}$ mice. In keeping with enhanced serum IL-6 concentrations, aging produced tissue-specific increases in zinc concentration of skeletal muscle and white adipose tissue. Metabolic endotoxemia produced by Zip14 ablation is maintained in aged $\mathrm{KO}$ mice. Muscle non-heme iron $(\mathrm{NHI})$ was increased in aged $\mathrm{WT}$ mice but not in aged Zip14 KO mice demonstrating NHI uptake by muscle is ZIP14-dependent and increases with age. NF-kB and STAT3 activation was greater in aged mice, but was tissue specific and inversely related to tissue zinc. Micro-CT analysis revealed that Zip14 KO mice had markedly reduced trabecular bone that was greatly amplified with aging. These results demonstrate that the inflammation-responsive zinc transporter ZIP14 has phenotypic effects that are amplified with aging. 


\section{Introduction}

Many mediators of the aging process are associated with inflammation and oxidative stress (Vasto et al., 2007; Frazzini et al., 2006). These consequences may be augmented if there is a concurrent low intake of zinc (Marcellini et al., 2006; Pepersack et al., 2001; Tudor et al., 2005). Proinflammatory conditions have been shown to influence zinc metabolism through cellular transport and intracellular zinc processing (Cousins et al., 2006). Many of the same mediators may influence aging. For example, it has been proposed that the proinflammatory cytokine IL-6 is a key factor in human age-associated diseases (Bennermo et al., 2004; Olivieri et al., 2006). A single nucleotide polymorphism (SNP) in the $I L-6$ gene is associated with an increased inflammatory response (Bennermo et al., 2004; Mocchegiani et al., 2008). IL-6 has been shown to regulate components of the homeostatic mechanisms for zinc. Specifically, IL-6 has been shown to influence metallothionein expression and cellular zinc accumulation (Schroeder and Cousins, 1990).

We have established that the zinc transporter ZIP14 (slc39a14), a zinc importer usually located at the cell surface, is regulated by proinflammatory cytokines, particularly IL-6 (Liuzzi et al., 2005). The regulation of Zip 14 by IL-6 suggests ZIP14 expression could be influenced by aging. Mice with a knockout (KO) of Zip14 have a phenotype that includes metabolic endotoxemia and altered glucose regulation (Aydemir et al., 2012; Guthrie et al., 2015; Troche et al., 2016). Such phenotypes are associated with aging. Here we present the first evidence on the influence of aging in mice with an ablation of Zip14. The aged mice were 22-24 months old thus placing them in an age comparable to humans between 56 and 69 years of age (Flurkey et al., 2007). The data shows that aging increases ZIP14 expression in muscle, that proinflammatory signaling pathways are influenced by aging and ZIP14 expression and that aging drastically reduced femoral trabecular bone with Zip14 ablation. 


\section{Materials and Methods}

\subsection{Mice}

Zip14 heterozygous mice of a C57BL/6J and 129SF1 mixed background were used as a breeding colony at the University of Florida (Aydemir et al., 2012). Both WT (Zip14 ${ }^{+/+}$) and

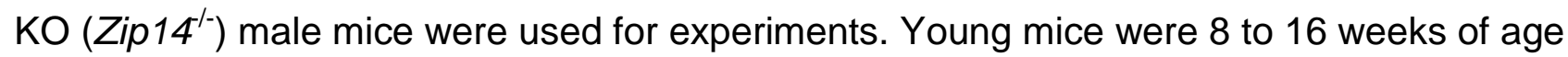
and aged mice were 22-24 months old. Based on experience with the Zip14 KO strain in terms of their proinflammatory status and their lower than normal body size, we decided to study the mice at 24 months of age. Mice were provided with commercial chow diet (Harlan 7912) and tap water ad libitum. Mice were anesthetized using isoflurane (Baxter, Deerfield, IL, USA) and euthanasia was via cardiac puncture and exsanguination. All murine protocols were approved by the University of Florida Institutional Animal Care and Use Committee.

\subsection{Serum and Tissue analyses}

Blood was collected in clot activator tubes (Capiject, Terumo Medical, Somerset, NJ, USA). Serum was obtained by centrifugation and stored at $-80^{\circ} \mathrm{C}$. Harvested tissues were snap-frozen in liquid nitrogen and stored at $-80^{\circ} \mathrm{C}$. Zinc concentrations were measured by flame atomic absorption spectrometry (AAS). Serum was diluted 1:5 in Milli-Q® water for AAS. Tissues were weighed and digested in $\mathrm{HNO}_{3}\left(90^{\circ} \mathrm{C}\right.$ for 3 hours) and diluted (1:1 to 1:5) with Milli-Q® water for AAS (Troche et al., 2016).

Tissue aliquots were homogenized in TRIzol reagent (Life Technologies, Thermo Fisher Scientific, Waltham, MA, USA) with a Bullet blender (Next Advance, Averill Park, NY, USA) using either zirconium oxide or stainless steel beads. Quantitative real-time PCR (qPCR) was performed using Express One-Step Superscriptmix (Invitrogen, Carlsbad, CA, USA) and a StepOnePlus Real-Time PCR detection system (Applied Biosystems, Foster City, CA, USA). 
121 TBP mRNA and GAPDH mRNA (muscle) were used to normalize qPCR data (Aydemir et al., 122 2012).

Tissue aliquots were homogenized as above in RIPA or non-denaturing lysis buffer 124 (Santa Cruz Biotechnology, Dallas, TX, USA) containing a protease/phosphatase inhibitor mix 125 (Thermo Fisher Scientific, Waltham, MA, USA) and phenylmethanesulfonyl fluoride (Sigma Aldrich, St. Louis, MO, USA). Proteins were separated using 10\% SDS-PAGE and transferred 127 to nitrocellulose with transfer verification through Ponceau Red staining. Protein abundance 128 was visualized by chemiluminesence (Super Signal West Pico, Thermo Fisher, Waltham, MA, 129 USA) and digital imaging (Protein Simple, San Jose, CA, USA). Tubulin was used as the gel 130 loading control. ZIP14 and ZIP8 polyclonal antibodies were produced in-house as previously 131 described (Ryu et al., 2008; Liuzzi et al., 2005). NF-kB, phosphorylated NF-kB, STAT3, 132 phosphorylated STAT3, ERK1,2, and phosphorylated ERK1,2 antibodies were purchased from 133 Cell Signaling Technology (Boston, MA, USA). Tissue non-heme iron was measured using 134 tissue homogenates by the ferrozine method (Rebouche, et al., 2004).

\subsection{Evaluation of bone structure with micro-CT}

Femurs from the left leg were removed and separated from adherent tissue and was stored at $-80^{\circ} \mathrm{C}$. Thawed bones were individually scanned using a Scano $\mu \mathrm{CT}$ scanner $(\mu \mathrm{CT}$ 40; Scano Medical AG, Bassersdorf, Switzerland) as described in detail by Cao and Picklo (2013). Cortical and trabecular bone of the mid-femur was analyzed.

\subsection{Statistical Analysis}

Reported data are as means \pm SE. Comparison of WT vs. KO genotype was by Student's $t$-test. Genotype and age designation were treated as independent effects. ANOVA analyses were performed using the PROC MIXED procedures of SAS version 9.2 (SAS 
144 Institute, Cary, NC, USA) and Prism InStat 3. Multiple treatment comparisons were analyzed using the Turkey-Kramer means separation, and significance was established at $P \leq 0.05$.

\section{Results}

3.1 Zinc concentrations of specific tissues are altered in aged mice

Zinc concentrations in spleens of WT mice were significantly decreased with age $(p<0.01)$. In contrast, zinc concentrations in WAT and skeletal muscle were significantly increased $(p<0.05)$ in aged mice (Table 1$)$. Brain zinc concentrations were also greater $(p<0.03)$ in the aged mice. The concentrations in other tissues including bone showed no significant differences, indicating tissue specificity in zinc accumulation with aging. Because of the potential for inflammation to influence spleen and muscle, we focused on these tissues using liver as the control.

3.2 Aging influenced serum zinc, endotoxin, IL-6 concentrations and spleen IL-6 mRNA as shown by body length (Supporting Fig. 1) and body weight (Fig. 1A). Of note is that Zip14 KO mice maintained body weight in contrast to WT mice. Muscle to body weight ratios of young male mice were less in the $\mathrm{KO}$ genotype, but were not measured in the aged mice. Serum endotoxin was significantly greater in the Zip $14 \mathrm{KO}$ mice. This signature of metabolic endotoxemia was maintained in the aged KO mice (Fig. 1B). Measurement of serum IL-6 at steady state, using a high sensitivity ELISA, revealed that the WT aged mice had greater levels than young WT mice. Aging greatly amplified the differential in serum IL-6 in Zip14 KO mice (Fig. 1C). The exact cellular source(s) of the elevated serum IL-6 is not known. It is of note that IL-6 mRNA levels in the spleen of the aged KO mice are double those of the other groups indicating splenic macrophages could be one site of origin of the elevated IL-6 (Fig. 167 1D). 
Activation of NF-KB, STAT3 and ERK1/2 was examined in spleen, muscle and liver of

170

171

172

173

174

175

176

177

178

179

180

181

182

183

184

185

186

187

188

189

190

191

192

young and aged WT and KO mice. Aging increased p-NF-KB in all tissues (Fig. 1E). This effect

was markedly less in muscle and liver of $\mathrm{KO}$ mice while it was amplified in spleen which may

lead to higher IL-6 expression. Aging also increased p-STAT3 in spleen and muscle, but

activation was not influenced by genotype. p-ERK1/2 was less in liver of aged mice (Fig. 1E),

but was not changed in the other tissues. Insulin receptor phosphorylation was less in aged

$\underline{\text { mice. }}$

3.4 Aging and ZIP14 expression influences tissue zinc and non-heme iron concentrations

Our initial hypothesis was that since serum IL-6 levels increase with age, expression of

Zip14, which is induced by IL-6 (Liuzzi et al., 2005), may increase with age enhancing zinc

transport and retention in specific tissues. A comparison of steady state serum zinc

concentrations revealed that these were significantly greater $(p<0.02)$ in the Zip14 KO mice

(Fig. 2A). There was no effect due to age. A comparison of tissue zinc levels in WT versus KO mice revealed significant $(p<0.01)$ increases in muscle of aged WT mice (Fig. 2B and C).

These levels were increased further in muscle of aged Zip14 KO mice (Fig. 2C). This suggests that zinc transport kinetics are differentially altered in a tissue-specific fashion with aging. $\mathrm{NHI}$ was not influenced by aging in liver. In contrast, however, $\mathrm{NHI}$ levels in muscle were influenced by aging in the Zip14 KO mice used for these experiments (Fig. 2D).

3.5 ZIP8 and ZIP14 are differentially expressed with aging

We examined ZIP14 and its closest homologue ZIP8 (Schmitt-Ulms et al., 2009) in tissues of young and aged WT and KO mice. Protein levels for ZIP14 in liver of WT mice were comparable (Fig. 3A). ZIP14 in muscle was much more abundant in aged mice (Fig. 3A). In contrast, muscle ZIP8 expression was greatly increased with aging (Fig. 3B). Liver ZIP8 levels were much greater in the Zip14 KO mice. Aging increased ZIP8 levels in WT and KO mice with 
193 aging. These data suggest multiple factors are involved regulation of these transporters with 194 aging. Of note is that MT1 mRNA levels were not influenced in muscle or spleen by aging or 195 genotype (Fig. 3C).

1963.6 Growth, cortical bone volume/surface area, and trabecular bone are altered with aging in Zip14 KO mice

Bone morphology was influenced by both age and Zip14 expression. Specifically, cortical bone volume decreased in aged KO mice and bone surface area increased in aged KO 200 mice (Fig. 4A and 4B). Both of these measurements indicated that Zip14 ablation reduced 201 bone mass. The striking loss of trabecular bone of the femur in mice with the null Zip14 202 mutation is shown in the $\mu \mathrm{CT}$ 3D representations (Fig. 5A). While not pronounced in young 203 Zip14 KO mice, the aged KO mice showed significant reductions in trabecular bone based on 204 numerical indices of bone mineral density (Fig. 5B). In keeping with these results Ear 205 Mesenchymal Stem Cells (EMSC) from Zip14 KO mice and cultured in osteogenic medium 206 were less mineralized than WT counterparts (Supporting Fig. 2). The effect of LPS while 207 dramatic in WT, was not significant in KO EMSC. Further evaluation of these cells through 208 western blots showed that pSmad1/5, a key transducer of the Bone morphogenetic protein 209 (BMP) pathway, was downregulated with KO.

\section{4. Discussion}

There is currently great interest in the relationship of zinc transporter activity and 212 disease. The range is extensive and has implicated many of the $24 \mathrm{ZnT} / \mathrm{ZIP}$ proteins that 213 transport zinc. It has been proposed that the large number of zinc transporters is necessitated 214 because of the plethora of intracellular functions in which this essential micronutrient 215 participates (Lichten and Cousins, 2009a). Examples for the ZIP transporters/disease 216 relationship include adipose inflammation and ZIP14 (Troche et al., 2016), ZIP8 and ZIP14 and 
217 response to sepsis (Knoell et al., 2009; Wessels and Cousins, 2015), ZIP13 and Ehlers-Danlos 218 syndrome (Jeong et al., 2012), ZIP14 and metabolic endotoxemia (Guthrie et al., 2015), ZIP8 219 and osteoarthritis (Kim et al., 2014) and mutant Zip14 and parkinsonism/dystonia (Tuschi et 220 al., 2016). One report that relates ZIP transporter function to aging was an evaluation of Zip6 221 mRNA expression in immune cells (Wong et al., 2013). The findings reported here are the first 222 to specifically target the phenotypes of a ZIP transporter that are accentuated with aging. skeletal muscle and bone/ connective tissues. In experiments reported here we compared the response of muscle to other tissues with respect to zinc homeostasis, proinflammatory signaling pathways and bone development. The animals in this study were at steady state and only males were compared. The aged mice used were $22-24$ months old and are comparable to humans between 56 and 69 years of age (Flurkey et al., 2007). This age range is appropriate because potential therapeutic intervention studies to combat age-related disease routinely use mice that are about 22 months old to test effectiveness (Camporez et al., 2016). Of the original cohort of WT and KO mice reserved for this study, no mortality was observed during the 22 month comparison period. Gross examination would however classify the KO $\underline{\text { mice as low-functioning compared to the WT mice. }}$ The Zip14 KO genotype clearly handles aspects of aging differently than WT mice. We interpret the increases of serum zinc levels in both young and aged Zip14 KO mice as indicative of a decrease in ZIP14-mediated uptake of systemic zinc. Tissue zinc levels in muscle are greater in aged mice, however. The finding that increased circulating IL-6, a signature of aging (Oliveri et al., 2006), is substantially increased in Zip14 KO mice suggests that the loss of ZIP14 leads to overexpression of IL-6 production, most likely through loss of a feedback loop. As mentioned earlier, the spleen could be a source of circulating IL-6 as IL-6 
241 mRNA is increased. Previously we have shown that ZIP14 regulates inflammatory signaling in

242 adipose tissue (Troche et al., 2016). Adipose is a major source of the increased IL-6

243 associated with aging (Starr et al., 2009). Hence in the Zip14 KO mice, adipose tissue may be

244 a contributor to increased systemic IL-6 observed with aging in our experiments here as this

245 genotype has increased body fat (Aydemir et al., 2012).

$246 \quad$ Elevated muscle zinc concentrations are in agreement with the increased ZIP14 in

247 muscle of WT mice. Greater levels of zinc in muscle of Zip14 KO mice suggest a

248 compensatory zinc transport function has occurred. The upregulation of muscle ZIP8 could

249 contribute to this increase. Aging clearly increased ZIP8 expression in muscle and also liver.

250 Most research on ZIP8 expression/function has focused on immune cells, starting with its

251 identification via inducibility by lipopolysaccharide (Begum et al., 2002). We identified that

252 activation of human primary T-cells dramatically increased ZIP8 and influenced intracellular

253 zinc and inhibition of calcineurin phosphatase activity (Aydemir et al., 2009). Migration of ZIP8

254 as distinct bands of $75 \mathrm{KDa}$ and $150 \mathrm{KDa}$ in western analysis were noted at that time. Multiple

255 molecular weights were noted here for splenic, muscle and liver ZIP8 (Fig 3D). The difference

256 in molecular weight of ZIP8 could be related to shedding of the ectodomains (Ehsani et al,

257 2011) or formation of a homo- or hetero-dimer. To our knowledge, the current experiments are

258 the first to report ZIP8 expression in skeletal muscle and the first to show up-regulation with

259 aging.

260 Muscle was selected as a focus for these studies because of the influence of aging and 261 sarcopenia and our previous profiling of Zip transporter mRNAs that respond to

262 proinflammatory stimuli. Following an LPS injection (18 hr) Zip14 mRNA was increased 18-fold 263 in skeletal muscle (Aydemir et al., 2012) and Zip8 mRNA with a 4-fold increase was the only 264 other Zip mRNA showing a major response to LPS treatment (unpublished data). This is of 
265 interest since ZIP14 and ZIP8 have the closest homology among the ZIP transporter family 266 and comprise one branch of the LIV-1 subfamily (Schmitt-Ulms et al., 2009). ZIP14 has been 267 localized to the plasma membrane and endosomes (Liuzzi et al., 2005; Guthrie et al., 2015 268 Troche et al., 2016) and ZIP8 has been detected at the plasma membrane, mitochondria and 269 lysosomes (He et al., 2006; Besecker et al., 2008; Aydemir et al., 2009). Under in vitro 270 conditions these ZIP transporters may facilitate the movement of zinc and in some cases iron, 271 manganese and cadmium (Lichten and Cousins, 2009). The magnitude of these metal fluxes is 272 likely dependent upon the available pool of free metal ion to serve as a substrate for transport. Our new findings merge with a concept where $\mathrm{NHI}$ accumulates in muscle and may 274 contribute to muscle decline-of-function and atrophy (Xu et al., 2010). We demonstrate here 275 that $\mathrm{NHI}$ does not accumulate in muscle of male Zip14 KO mice as it does in male WT mice 276 (Fig. 2D). This suggests ZIP14 is responsible for the NHI accumulation. Aging male mice show 277 augmented ZIP14 and ZIP8 in muscle as shown in this report (Fig $3 \mathrm{~A}, \mathrm{~B}$ ). ZIP8 can also 278 transport iron in vitro, albeit to a more limited extent than zinc (Wang et al., 2012). The 279 prevention of muscle $\mathrm{NHI}$ accumulation with Zip14 ablation suggests ZIP8 is not a factor in 280 muscle $\mathrm{NHI}$ accumulation. In contrast, previously we observed that ablation of Zip14 increased $281 \mathrm{NHI}$ in liver of young Zip14 KO female mice (Aydemir et al., 2012). Collectively these findings suggest that accumulation of $\mathrm{NHI}$ in muscle and liver in mice is influenced by both gender and age. The data support the concept that gender differences in iron metabolism occur in murine 284 models.

Recent studies have clearly established a link between murine osteoarthritis, zinc and ZIP8 (Kim et al., 2014). In this scenario, zinc increases MTF-1 activation and thereby upregulated expression of specific metalloproteases involved in bone matrix degradation. Most likely a comparable situation is not involved in the current experiments as Mt-1 mRNA 
expression did not differ as a function of age in mice of the Zip14 KO genotype. Mt-1 mRNA is a sentinel for MTF-1 activation. We have focused here on muscle and spleen. Aging may produce changes in labile zinc pools in specific cells, e.g. leukocytes, Approaches to address such issues directly are beyond the scope of this report.

The influence of aging on femoral bone density in the aged mice observed in our studies by several indices using micro-CT is in line with age-related bone loss in humans (Khosla, 2013). The mice in this study were males eliminated from our breeding colony and allowed to age. At 22-24 months of age they are comparable to humans aged 56-69 years (Flurkey et al., 2007). Of major emphasis is the marked reduction in trabecular bone in only the aged KO mice. The mechanism responsible is not currently established. ZIP14 could influence bone accretion or resorption through zinc regulated signaling systems. This could include a role for IL-6. Our preliminary finding with osteogenic differentiation of EMSC from WT and Zip14 KO mice would support that concept (Supporting Fig. 2). Upregulation of NF-KB activation in stem cells derived from the Zip14 KO mice supports that motion. We cannot rule out the involvement of another ZIP14-regulated process in the loss of trabecular bone in the aged Zip14 KO mice. It has been postulated, based on in vitro transfection studies and data from zebrafish, that the major role for ZIP14 is to transport Mn (Tuschl et al., 2016). If that hypothesis is correct, ZIP14 dysfunction could reduce biliary Mn elimination, causing Mn retention in the brain leading to neurological disorders. Nutritional Mn deficiency has been shown to produce skeletal abnormalities in animals however excess Mn has not been shown to influence bone (Underwood, 1977). Hence Mn deficiency or toxicity is not a likely factor in the loss of trabecular bone observed in the aged Zip14 KO mice as described in our report.

\section{Conclusion}


In summary, the data presented in this report show that aging influences zinc

313 metabolism particularly in muscle (Fig. 6). This suggests some influence of proinflammatory

314 cytokines and related signaling pathways. Activation of NF-KB and STAT3 pathways in muscle

315 is most likely involved. Ablation of ZIP14 accentuates the elevated circulating levels of

316 proinflammatory IL-6 and produces metabolic endotoxemia. Increases in ZIP8 expression

317 suggest this transporter is also dysregulated in aging. ZIP14 ablation eliminates the NHI

318 accumulation in muscle that occurs in aging. Proper functioning of ZIP14 in bone is essential

319 during aging to maintain trabecular and cortical bone density. ZIP14 may influence diseases of 320 aging including sarcopenia and osteoporosis. 


\section{Acknowledgments}

323

The authors are grateful to Dr. Jay Cao of the USDA Grand Forks Human Nutrition

324 Research Center for performing the micro CT scans of the bone and the resulting analyses.

325 We also thank Matthew P. Beke for help with the manuscript.

326 Funding

327 This project was supported by the National Institute of Diabetes and Digestive and 328 Kidney Diseases (Grant No R01-DK094244) and Boston Family Endowment Funds of the 329 University of Florida Foundation to RJC.

330 Author contributions

T.B.A., C.T. and R.J.C. designed the experiments. T.B.A., C.T., J.K., M.H.K., and O.T. 332 performed the experiments; T.B.A., C.T., C.L. and R.J.C. contributed to data interpretation and 333 T.B.A. and R.J.C. wrote the paper. All authors critically reviewed and gave final approval to this 334 version of the manuscript.

335 Conflict of Interest

$336 \quad$ The authors have no conflict of interest. 


\section{References}

Aydemir TB, Chang SM, Guthrie GJ, Maki AB, Ryu MS, Karabiyik A, Cousins RJ (2012). Zinc transporter ZIP14 functions in hepatic zinc, iron and glucose homeostasis during the innate immune response (endotoxemia). PloS one. 7, e48679.

Aydemir TB, Liuzzi JP, McClellan S, Cousins RJ (2009). Zinc transporter ZIP8 (SLC39A8) and zinc influence IFN-y expression in activated human T cells. J Leuk Biol. 86, 337-348.

Begum NA, Kobayashi M, Moriwaki Y, Matsumoto M, Toyoshima K, Seya T (2002). Mycobacterium bovis BCG cell wall and lipopolysaccharide induce a novel gene, BIGM103, encoding a 7-TM protein: identification of a new protein family having $\mathrm{Zn}$ transporter and Zn-metalloprotease signatures. Genomics. 80, 630-645.

Bennermo M, Held C, Stemme S, Ericsson CG, Silveira A, Green F, Tornvall P (2004). Genetic predisposition of the interleukin-6 response to inflammation: implications for a variety of major diseases? Clin chem. 50, 2136-2140.

Besecker B, Bao S, Bohacova B, Papp A, Sadee W, Knoell DL (2008). The human zinc transporter SLC39A8 (Zip8) is critical in zinc-mediated cytoprotection in lung epithelia. Am J Physiol Lung Cell Mol Physiol. 294, L1127-1136.

Camporez JP, Petersen MC, Abudukadier A, Moreira GV, Jurczak MJ, Friedman G, Haqq CM, Petersen KF, Shulman GI (2016). Anti-myostatin antibody increases muscle mass and strength and improves insulin sensitivity in old mice. Proc Natl Acad Sci U S A. 113, 2212-2217.

Cao JJ, Picklo MJ (2014). N-acetylcysteine supplementation decreases osteoclast differentiation and increases bone mass in mice fed a high-fat diet. J Nutr. 144, 289296. 
367 Cousins RJ, Liuzzi JP , Lichten LA (2006). Mammalian zinc transport, trafficking, and signals. J Biol Chem. 281, 24085-24089.

Ehsani S, Huo H, Salehzadeh A, Pocanschi CL, Watts JC, Wille H, Westaway D, Rogaeva E, St George-Hyslop PH, Schmitt-Ulms G (2011). Family reunion--the ZIP/prion gene family. Prog Neurobiol. 93, 405-420.

Fagiolo U, Cossarizza A, Scala E, Fanales-Belasio E, Ortolani C, Cozzi E, Monti D, Franceschi C, Paganelli R (1993). Increased cytokine production in mononuclear cells of healthy elderly people. Eur J Immunol. 23, 2375-2378.

Flurkey K, M. Currer J, Harrison DE (2007). Chapter 20 - Mouse Models in Aging Research A2 - Fox JG. In The Mouse in Biomedical Research (Second Edition). (MT Davisson, FW Quimby, SW Barthold, CE Newcomer, AL Smith, eds). Burlington: Academic Press, pp. 637-672.

Frazzini V, Rockabrand E, Mocchegiani E, Sensi SL (2006). Oxidative stress and brain aging: is zinc the link? Biogerontology. 7, 307-314.

Guthrie GJ, Aydemir TB, Troche C, Martin AB, Chang SM, Cousins RJ (2015). Influence of ZIP14 (slc39A14) on intestinal zinc processing and barrier function. Am J Physiol Gastrointest Liver Physiol. 308, G171-178.

He L, Girijashanker K, Dalton TP, Reed J, Li H, Soleimani M, Nebert DW (2006). ZIP8, member of the solute-carrier-39 (SLC39) metal-transporter family: characterization of transporter properties. Mol Pharmacol. 70, 171-180.

Ionescu AM, Schwarz EM, Vinson C, Puzas JE, Rosier R, Reynolds PR, O'Keefe RJ (2001). PTHrP modulates chondrocyte differentiation through AP-1 and CREB signaling. J Biol Chem. 276, 11639-11647. 
390 Jeong J, Walker JM, Wang F, Park JG, Palmer AE, Giunta C, Rohrbach M, Steinmann B, Eide

DJ (2012). Promotion of vesicular zinc efflux by ZIP13 and its implications for spondylocheiro dysplastic Ehlers-Danlos syndrome. Proc Natl Acad Sci U S A. 109, E3530-3538.

Khosla S (2013). Pathogenesis of age-related bone loss in humans. J Gerontol A Biol Sci Med Sci. $68,1226-1235$.

Kim JH, Jeon J, Shin M, Won Y, Lee M, Kwak JS, Lee G, Rhee J, Ryu JH, Chun CH, Chun JS (2014). Regulation of the catabolic cascade in osteoarthritis by the zinc-ZIP8-MTF1 axis. Cell. 156, 730-743.

Knoell DL, Julian MW, Bao S, Besecker B, Macre JE, Leikauf GD, DiSilvestro RA , Crouser ED (2009). Zinc deficiency increases organ damage and mortality in a murine model of polymicrobial sepsis. Crit Care Med. 37, 1380-1388.

Lichten L, Cousins RJ (2009). Mammalian zinc transporters: nutritional and physiologic regulation. Annu Rev Nutr. 29, 153-176.

Lichten LA, Liuzzi JP, Cousins RJ (2009). Interleukin-1 beta contributes via nitric oxide to the upregulation and functional activity of the zinc transporter Zip14 (Slc39a14) in murine hepatocytes. Am J Physiol Gastrointest Liver Physiol. 296, G860-867.

Liuzzi JP, Lichten LA, Rivera S, Blanchard RK, Aydemir TB, Knutson MD, Ganz T, Cousins RJ (2005). Interleukin-6 regulates the zinc transporter Zip14 in liver and contributes to the hypozincemia of the acute-phase response. Proc Natl Acad Sci U S A. 102, 6843-6848. Marcellini F, Giuli C, Papa R, Gagliardi C, Dedoussis G, Herbein G, Fulop T, Monti D, Rink L, Jajte J, Mocchegiani E (2006). Zinc status, psychological and nutritional assessment in old people recruited in five European countries: Zincage study. Biogerontology. 7, 339345. 
414 Mocchegiani E, Malavolta M (2008). Zinc-gene interaction related to inflammatory/immune response in ageing. Genes Nutr. 3, 61-75.

416

417

418

419

420

421

422

423

424

425

426

427

428

429

430

431

432

433

434

435

436

437

Olivieri F, Antonicelli R, Cardelli M, Marchegiani F, Cavallone L, Mocchegiani E, Franceschi C (2006). Genetic polymorphisms of inflammatory cytokines and myocardial infarction in the elderly. Mech Ageing Dev. 127, 552-559.

Pepersack T, Rotsaert P, Benoit F, Willems D, Fuss M, Bourdoux P, Duchateau J (2001). Prevalence of zinc deficiency and its clinical relevance among hospitalised elderly. Arch Gerontol Geriatr. 33, 243-253.

Rebouche CJ, Wilcox CL, Widness JA (2004). Microanalysis of non-heme iron in animal tissues. J Biochem Biophys Methods. 58, 239-251.

Ryu MS, Lichten LA, Liuzzi JP, Cousins RJ (2008). Zinc transporters ZnT1 (Slc30a1), Zip8 (Slc39a8), and Zip10 (Slc39a10) in mouse red blood cells are differentially regulated during erythroid development and by dietary zinc deficiency. J Nutr. 138, 2076-2083.

Schmitt-Ulms G, Ehsani S, Watts JC, Westaway D, Wille H (2009). Evolutionary descent of prion genes from the ZIP family of metal ion transporters. PloS one. 4, e7208.

Schroeder JJ, Cousins RJ (1990). Interleukin 6 regulates metallothionein gene expression and zinc metabolism in hepatocyte monolayer cultures. Proc Natl Acad Sci U S A. 87, 31373141.

Starr ME, Evers BM, Saito H (2009). Age-associated increase in cytokine production during systemic inflammation: adipose tissue as a major source of IL-6. J Gerontol A Biol Sci Med Sci. 64, 723-730.

Troche C, Beker Aydemir T, Cousins RJ (2016). Zinc transporter Slc39a14 regulates inflammatory signaling associated with hypertrophic adiposity. Am J Physiol Endocrinol Metab. 310, E258-268. 
438 Tudor R, Zalewski PD, Ratnaike RN (2005). Zinc in health and chronic disease. J Nutr Health Aging. 9, 45-51.

440 Tuschl K, Meyer E, Valdivia LE, Zhao N, Dadswell C, Abdul-Sada A, Hung CY, Simpson MA, 441 Chong WK, Jacques TS, Woltjer RL, Eaton S, Gregory A, Sanford L, Kara E, Houlden H, Cuno SM , Prokisch H (2016). Mutations in SLC39A14 disrupt manganese homeostasis and cause childhood-onset parkinsonism-dystonia. Nat Commun 7, 11601.

Underwood, ER (1977). Manganese in: Trace Elements in Human and Animal Nutrition, fourth edition. New York: Academic Press.

Vasto S, Candore G, Balistreri CR, Caruso M, Colonna-Romano G, Grimaldi MP, Listi F, Nuzzo D, Lio D , Caruso C (2007). Inflammatory networks in ageing, age-related diseases and longevity. Mech Ageing Dev. 128, 83-91.

Wang CY, Jenkitkasemwong S, Duarte S, Sparkman BK, Shawki A, Mackenzie B, Knutson MD (2012). ZIP8 is an iron and zinc transporter whose cell-surface expression is upregulated by cellular iron loading. J Biol Chem. 287, 34032-34043.

Wessels I, Cousins RJ (2015). Zinc dyshomeostasis during polymicrobial sepsis in mice involves zinc transporter Zip14 and can be overcome by zinc supplementation. $A m \mathrm{~J}$ Physiol Gastrointest Liver Physiol. 309, G768-778.

Wong CP, Magnusson KR, Ho E (2013). Increased inflammatory response in aged mice is associated with age-related zinc deficiency and zinc transporter dysregulation. J Nutr Biochem. 24, 353-359.

Xu J, Marzetti E, Seo AY, Kim JS, Prolla TA, Leeuwenburgh C (2010). The emerging role of iron dyshomeostasis in the mitochondrial decay of aging. Mech Ageing Dev. 131, 487493. 


\section{Supporting Information}

462 Supporting Fig. 1. Influence of aging on body length of WT and Zip14 KO mice.

Supporting Fig. 2. Ear mesenchymal stem cells (EMSC) were collected from WT and KO mice 465 ( $n=3$ mice per genotype) and processed as described previously (Rim et al., 2005; Troche et 466

al., 2016). Post-processing cells were plated and grown until confluence. Upon visual

confirmation of confluence cells were treated with osteogenic differentiation medium (DMEM-

F12 supplemented with $10 \%$ FBS, $200 \mu \mathrm{M}$ ascorbic acid 2-phosphate, 10nM dexamethasone, $7 \mathrm{mM} \beta$-glycerophosphate, and 1\% pencillin-streptomycin). Cells were maintained in culture for 18 days. For experiments that involved LPS, cells were maintained in osteogenic medium supplemented with $10 \mathrm{ng} / \mathrm{ml}$ LPS throughout the entire course of the experiment; medium was changed every 3 days.

Mineralization, a marker of osteogenic differentiation, was evaluated through Alizarin Red S (ARS) a compound that binds ionic calcium. At 14 days, cells were fixed in $4 \%$ formaldehyde and stained with $40 \mathrm{mM}$ ARS for $30 \mathrm{~min}$. Cells were washed, and images were taken. Elution of Alizarin Red S was achieved through a protocol previously described by Gregory et al. (2004). Briefly, previously stained cells were incubated in 10\% acetic acid for $30 \mathrm{~min}$ at room temperature. The monolayer was then scrapped from wells and heated at $85^{\circ} \mathrm{C}$ for $10 \mathrm{~min}$. Cells were pelleted by centrifugation and the supernatant was transferred to a new tube where $10 \%$ ammonium hydroxide was used to stop the reaction. Absorbance was red at $405 \mathrm{~nm}$.

\section{Supporting References}

Rim J-S, Mynatt RL, Gawronska-Kozak B (2005). Mesenchymal stem cells from the outer ear: a novel adult stem cell model system for the study of adipogenesis. FASEB J. 9, 12051207.

Gregory CA, Gunn WG, Peister A, Prockop DJ (2004). An Alizarin red-based assay of 
mineralization by adherent cells in culture: comparison with cetylpyridinium chloride extraction. Anal Biochem. 329, 77-84. 
Table 1

491

Zinc Concentrations of specific tissues of young (3-4 month) and aged (22 month) WT mice.

\begin{tabular}{|c|c|c|c|}
\hline Tissue & Age & $\mu \mathrm{g} \mathrm{Zn/mg} \mathrm{tissue}$ & P-Value \\
\hline \multirow[t]{2}{*}{ Spleen } & Young & $23.89 \pm 0.26$ & 0.01 \\
\hline & Aged & $22.10 \pm 0.47$ & \\
\hline \multirow[t]{2}{*}{ Adipose } & Young & $2.11 \pm 0.13$ & 0.04 \\
\hline & Aged & $2.73 \pm 0.16$ & \\
\hline \multirow[t]{2}{*}{ Muscle } & Young & $8.31 \pm 1.27$ & 0.05 \\
\hline & Aged & $12.35 \pm 1.27$ & \\
\hline \multirow[t]{2}{*}{ Brain } & Young & $5.52 \pm 0.11$ & 0.03 \\
\hline & Aged & $5.97 \pm 0.11$ & \\
\hline \multirow[t]{2}{*}{ Liver } & Young & $34.80 \pm 1.36$ & 0.4 \\
\hline & Aged & $34.75 \pm 1.13$ & \\
\hline \multirow[t]{2}{*}{ Heart } & Young & $13.95 \pm 1.10$ & 0.1 \\
\hline & Aged & $16.63 \pm 2.41$ & \\
\hline \multirow[t]{2}{*}{ Lung } & Young & $17.18 \pm 1.45$ & 0.4 \\
\hline & Aged & $16.90 \pm 1.34$ & \\
\hline \multirow[t]{2}{*}{ Pancreas } & Young & $29.89 \pm 0.31$ & 0.2 \\
\hline & Aged & $27.71 \pm 2.28$ & \\
\hline \multirow[t]{2}{*}{ Jejunum } & Young & $28.00 \pm 0.99$ & 0.1 \\
\hline & Aged & $26.56 \pm 1.00$ & \\
\hline \multirow[t]{2}{*}{ Cecum } & Young & $16.41 \pm 1.16$ & 0.3 \\
\hline & Aged & $17.13 \pm 0.86$ & \\
\hline \multirow[t]{2}{*}{ Colon } & Young & $23.65 \pm 1.55$ & 0.1 \\
\hline & Aged & $21.05 \pm 1.09$ & \\
\hline
\end{tabular}

492

Zinc was measured by AAS.

493

494

495

496

497 


\section{Figure Legends}

499 Fig. 1. Influence of aging and Zip14 ablation using young and aged WT and Zip14 KO mice on 500 (A) body weight, (B) serum endotoxin, (C) serum IL-6, (D) splenic IL-6 mRNA and (E) 501 inflammatory pathways in spleen, muscle and liver. (A)-(D) values are mean $\pm S E, n=4$ per 502 group. $\mathrm{P}<$ values are shown.

503 Fig. 2. Influence of aging and Zip14 ablation using young and aged WT and Zip14 KO mice on

504 (A) serum zinc concentration, (B) splenic zinc concentration, (C) muscle and liver zinc 505 concentrations, (D) muscle and liver non-heme iron (NHI) concentrations. Values are mean \pm 506 SE, $\mathrm{n}=4$ per group. $\mathrm{P}<$ values are shown.

507 Fig. 3. Influence of aging and Zip14 ablation using young and aged WT and Zip14 KO mice on

508 (A) muscle and liver ZIP14 using western blots normalized to tubulin, (B) spleen, muscle and 509 liver ZIP8 using western blots normalized to tubulin (C) MT1 mRNA in muscle and spleen. 510 Values are mean $\pm S E, n=4$ per group.

511 Fig. 4. Cortical bone microstructure of midshaft femur of young and aged WT and Zip14 KO 512 mice measured using micro-CT. (A) Representative 3D reconstructions of femur cross 513 sections. (B) Estimates of cortical bone volume/total volume (BV/TV), bone surface/bone 514 volume (BS/BV) and cortical thickness (TbTh). Values are mean $\pm \mathrm{SE}, \mathrm{n}=4$ per group.

515 Fig. 5. Trabecular bone microstructure of distal femur of young and aged WT and Zip14 KO 516 mice measured using micro-CT. (A) Representative 3D reconstructions of trabecular 517 architecture of the femur. (B) Estimates of trabecular bone volume/total volume (BV/TV), 518 connectivity density (ConnD), trabecular thickness $\mathrm{mgHA} / \mathrm{cm}^{3}(\mathrm{TbTh})$ and bone mineral density 519 (BMD). Values are mean $\pm \mathrm{SE}, \mathrm{n}=4$ per group. 
520 Fig. 6. Summary of changes associated with aging and those associated with ablation of 521 Zip14. Increased circulating IL-6 is a signature of aging that is augmented with ZIP14 ablation. 522 Aging increases muscle zinc and non-heme iron and decreases bone mineral density. These 523 are influenced with ablation of ZIP14. Metabolic endotoxemia is a signature of ZIP14 ablation, 524 but is not changed in aging. 


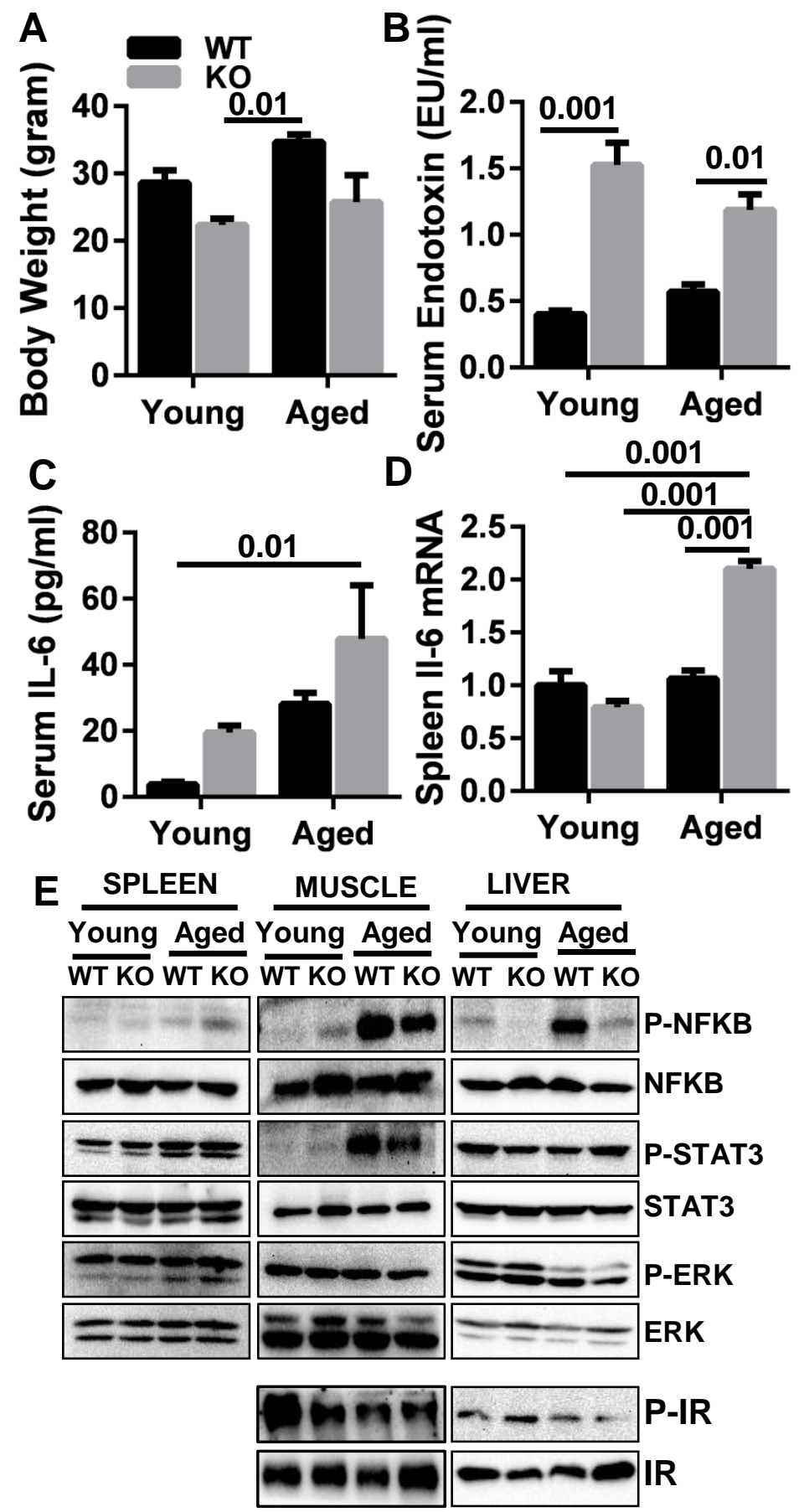



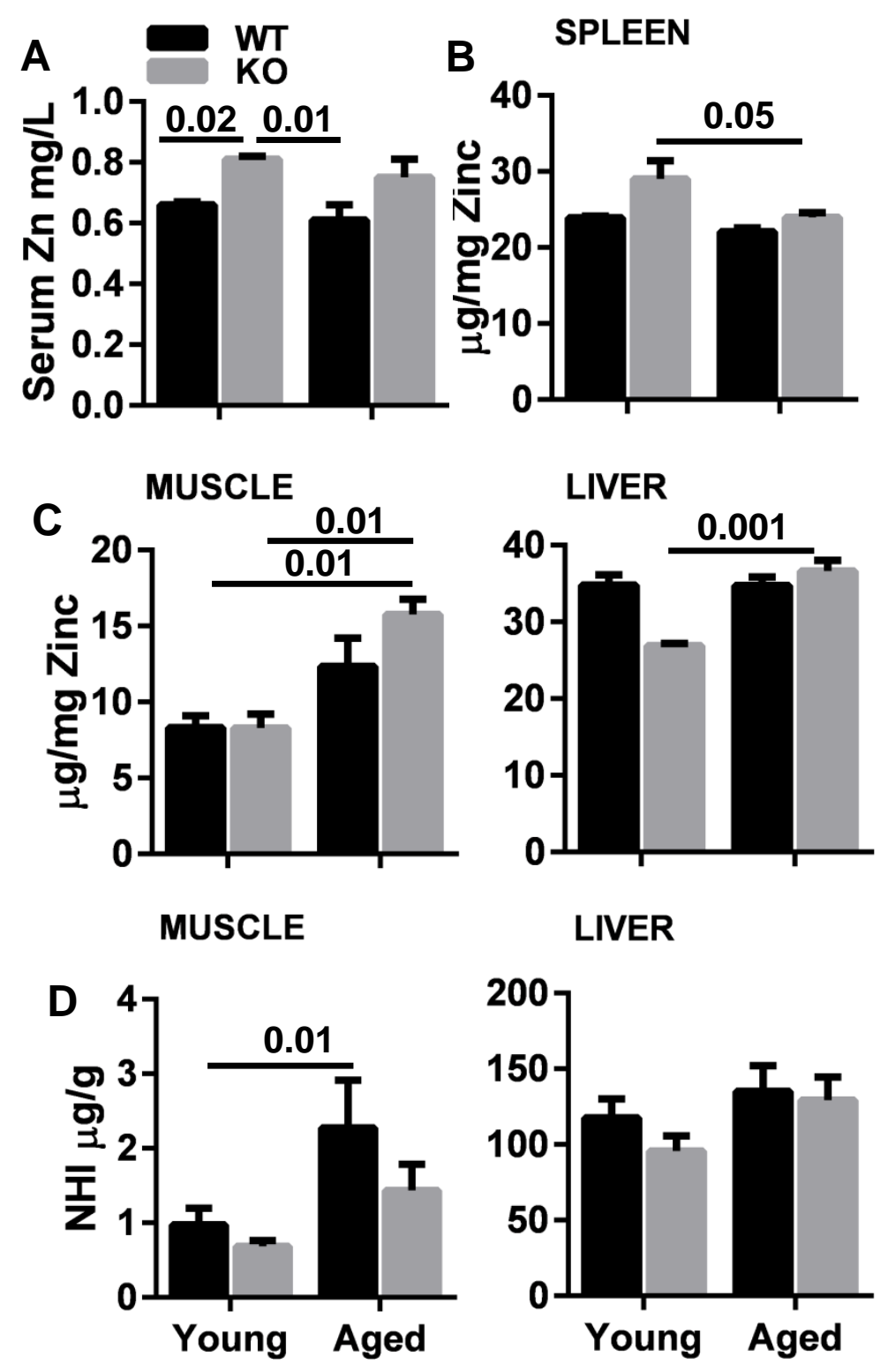
A MUSCLE LIVER

YOUNG AGED YOUNG AGED

FIGURE 3

WT KO WT KO WT KO WT KO

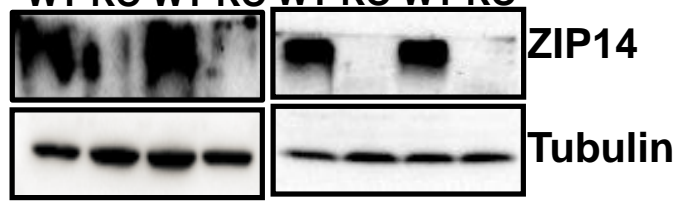

B SPLEEN MUSCLE LIVER

YOUNG AGED YOUNG AGED YOUNG AGED

WT KO WT KO WT KO WT KO WT KO WT KO
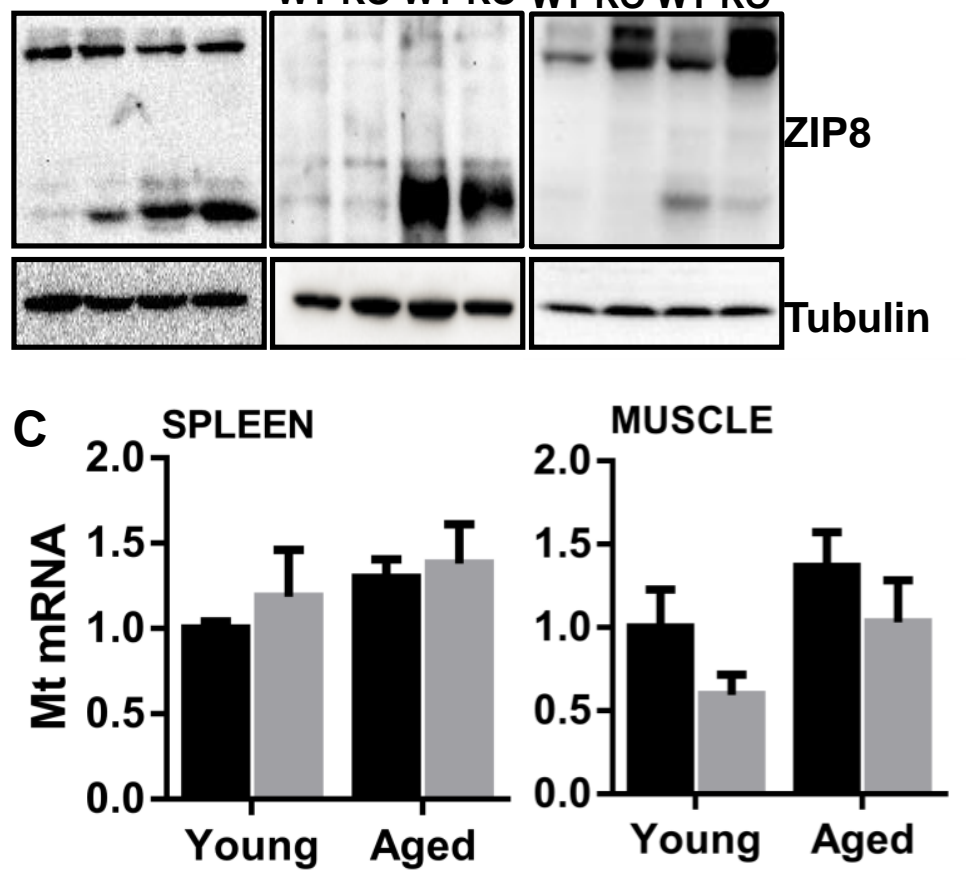


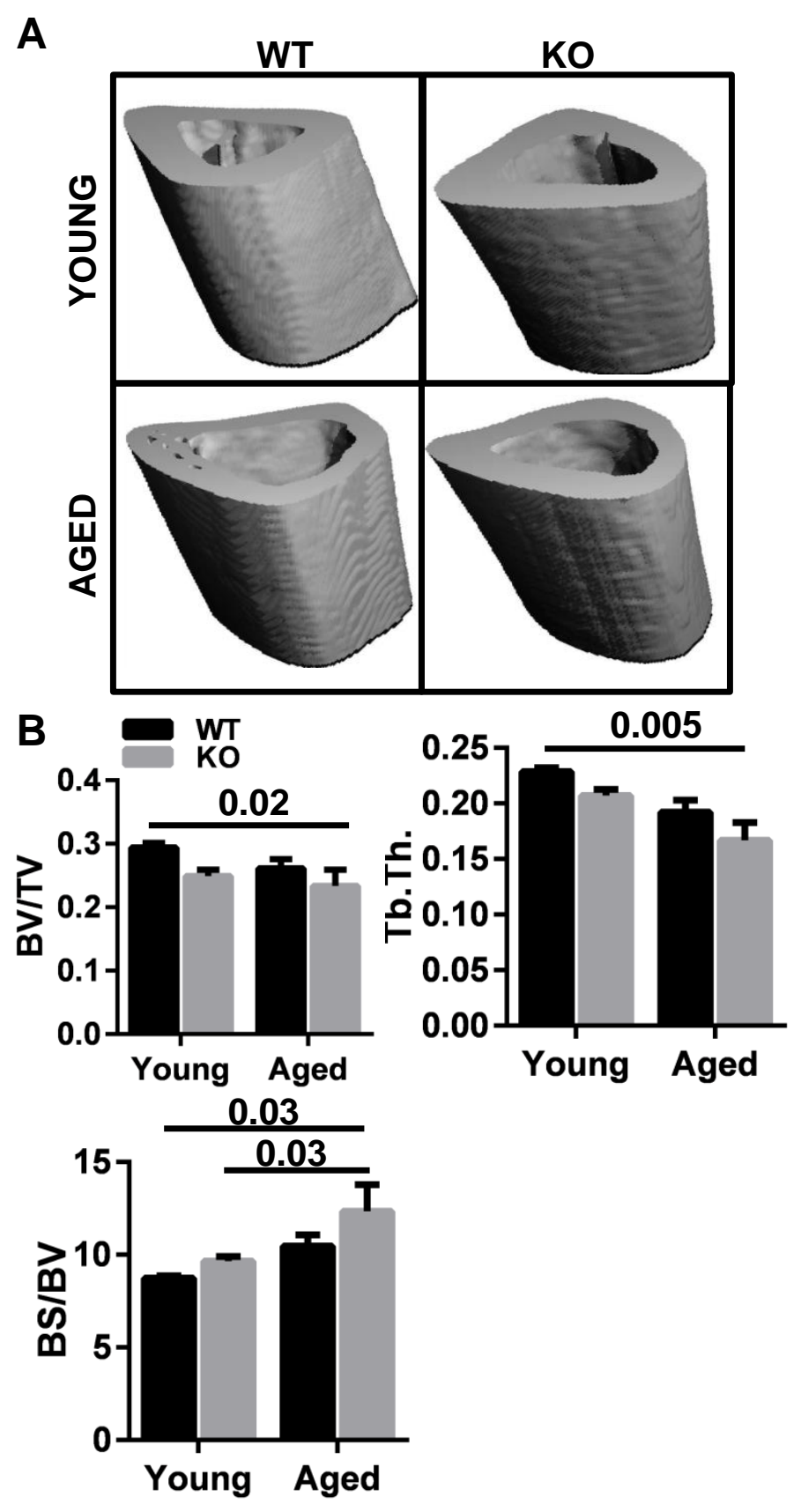

FIGURE 4 


\section{FIGURE 5}

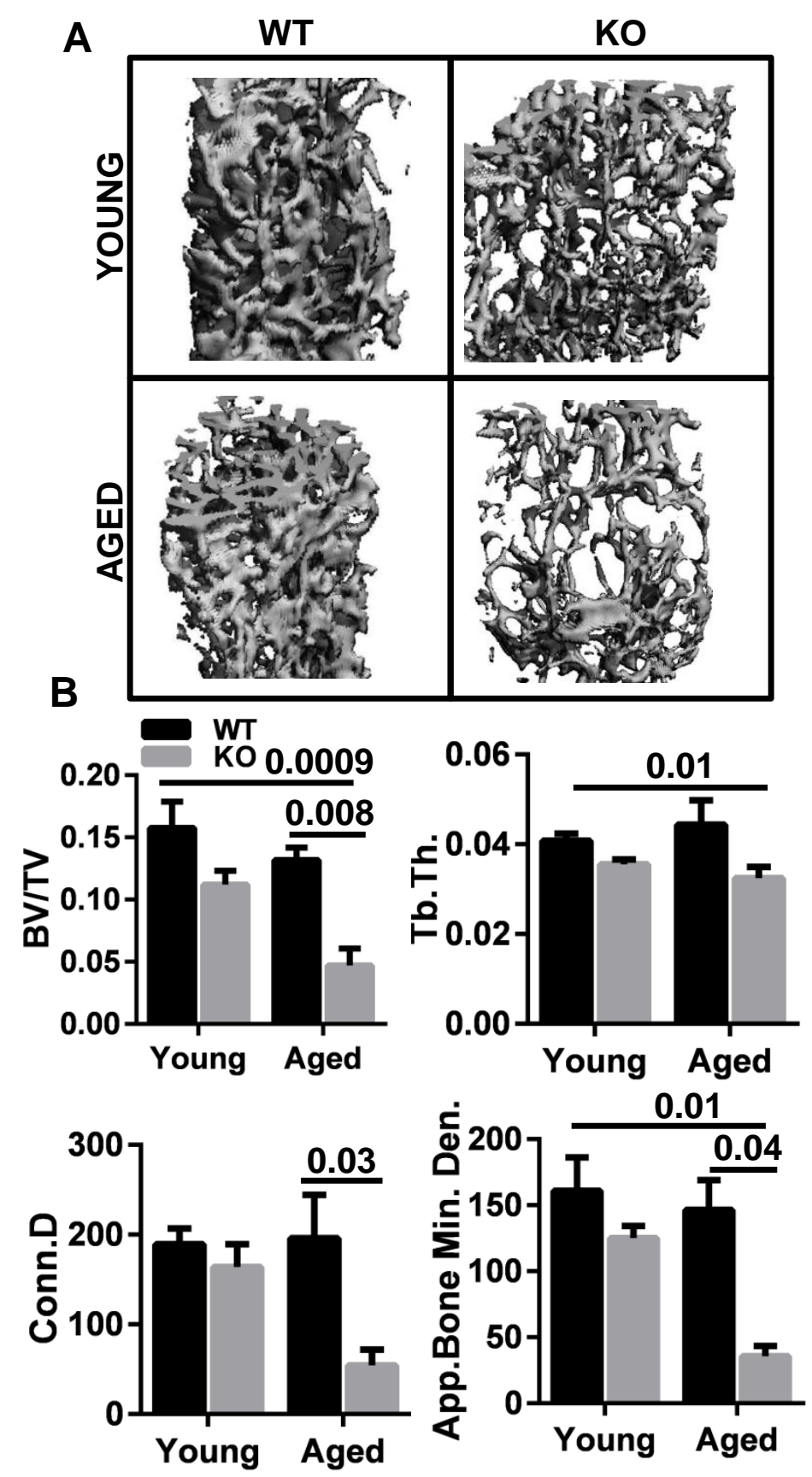


FIGURE 6

ZIP14 Deletion

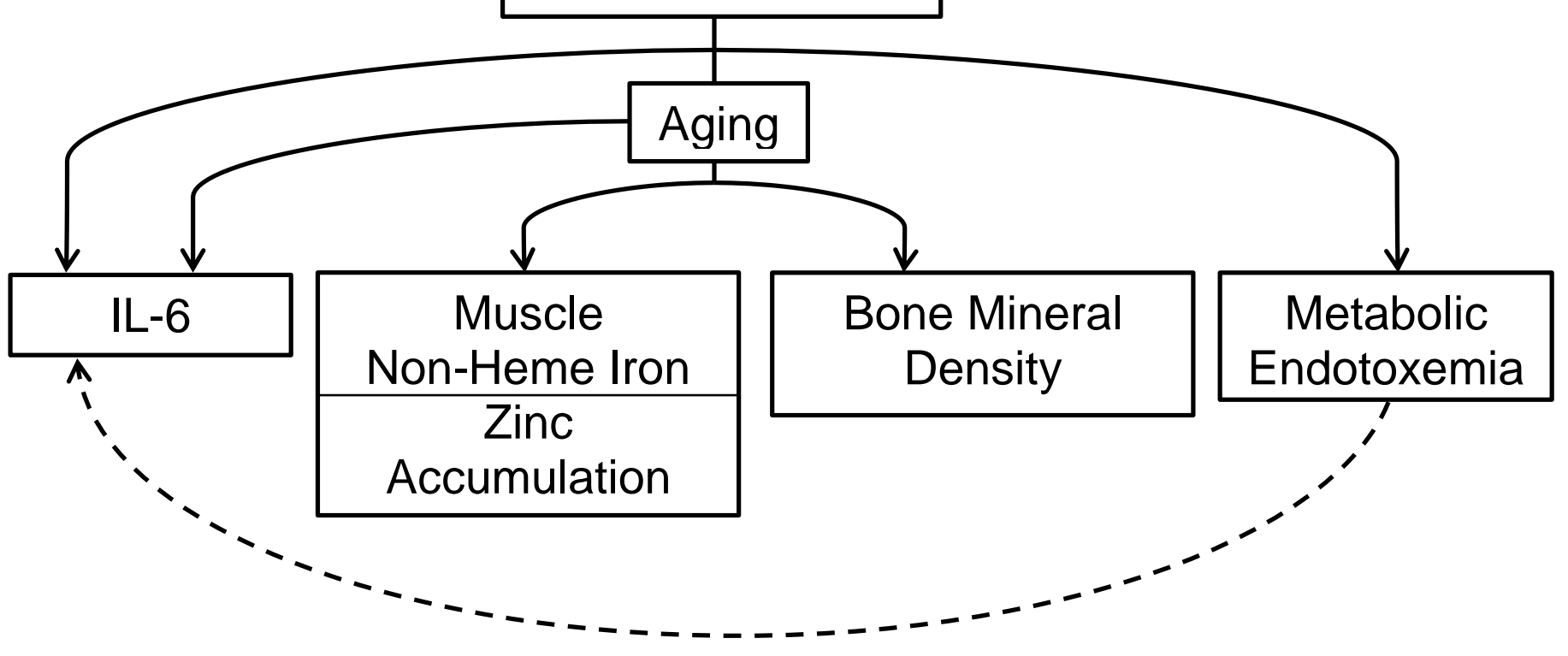




\section{Supporting Figure1}

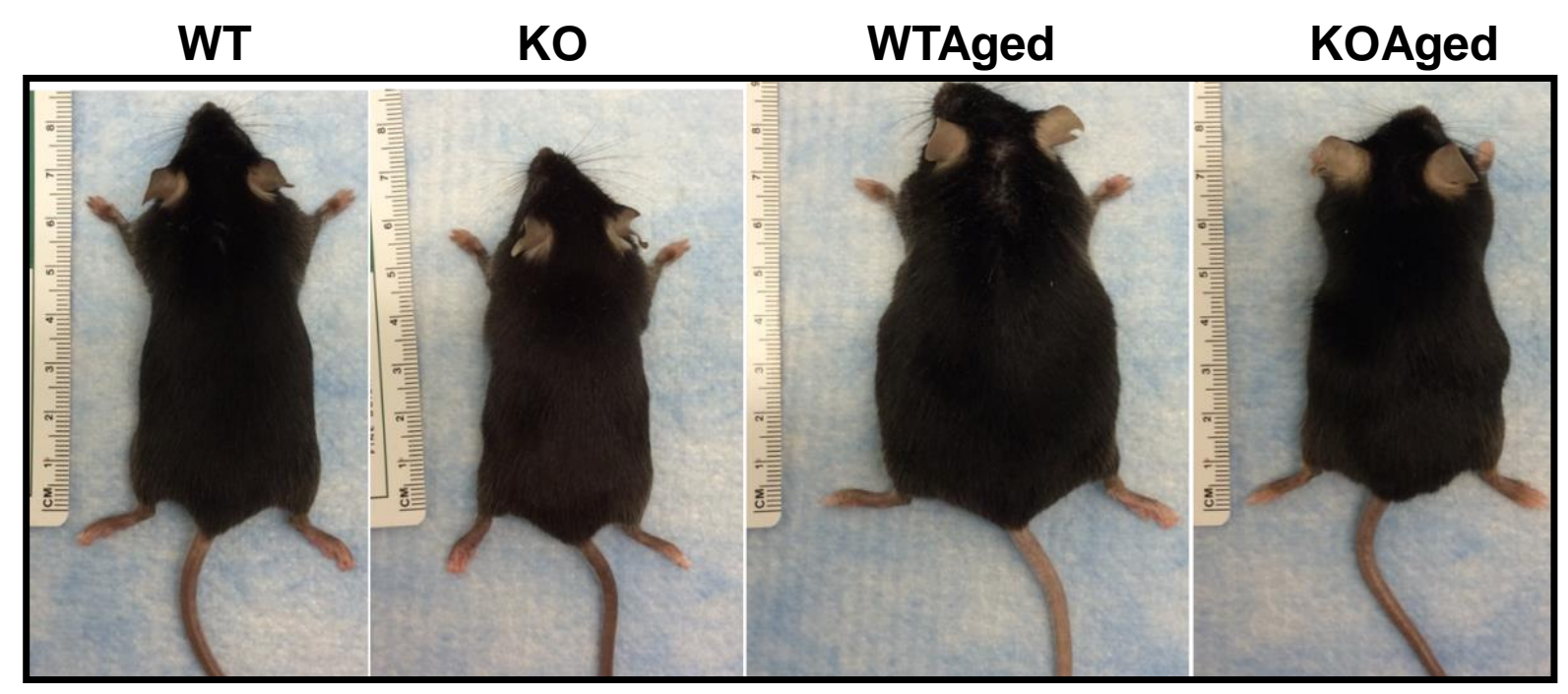




\section{Supporting Figure 2}
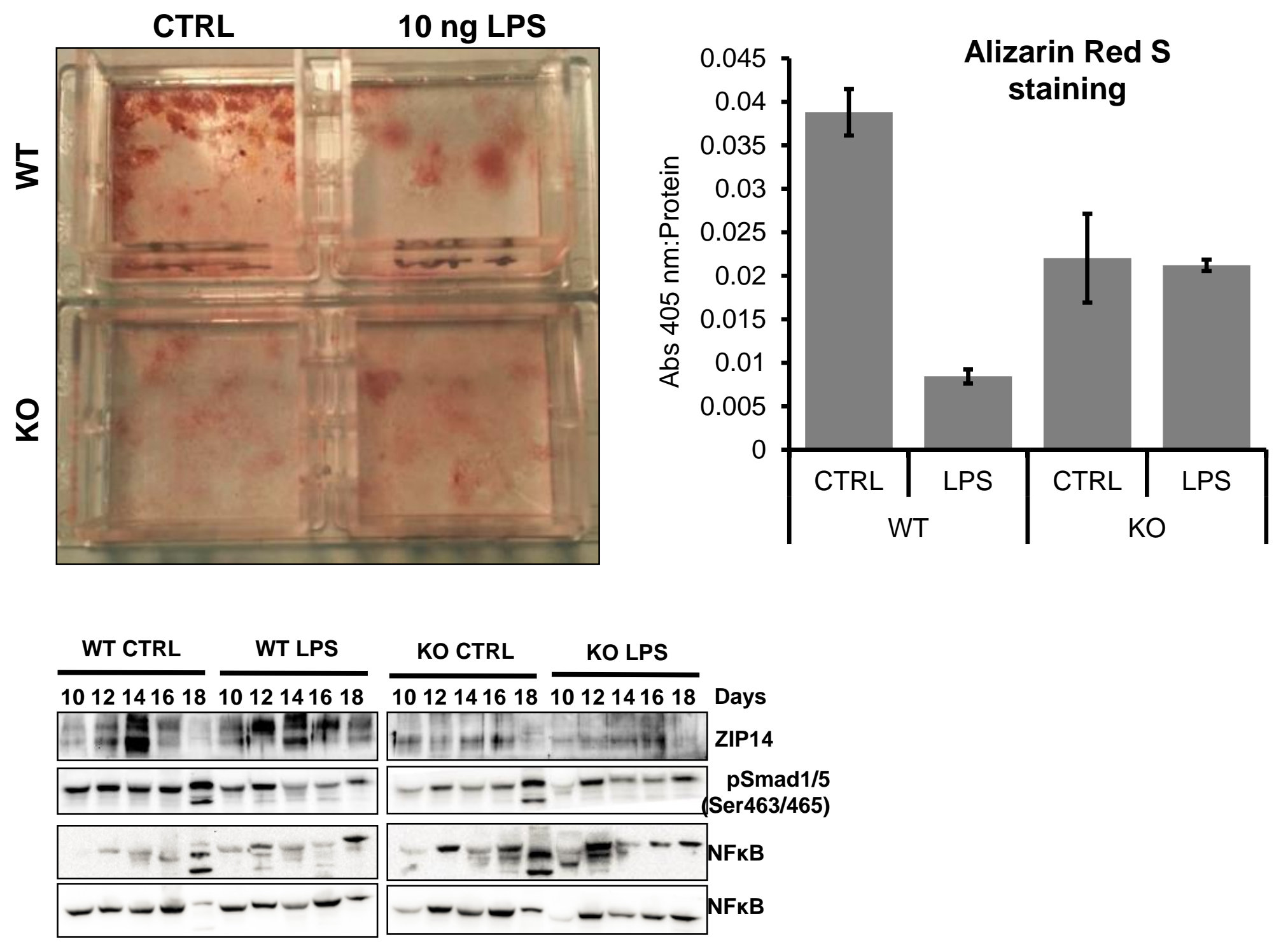